\title{
Guías de práctica clínica basadas en evidencia: Desarrollo y aplicación en neurología clínica en el Perú
}

\author{
Evidence based guidelines: Development and application in clinical neurology in Peru.
}

Mario Cornejo-Olivas ${ }^{1,2,7, a, b, c,}$, Carlos Alva-Diaz ${ }^{3,4,5, b, c,}$, Nilton Custodio Capuñay ${ }^{6, b, c ; ~ 7, d ~}$

Las guías de práctica clínica basadas en evidencia (GPC) son recomendaciones sistemáticamente revisadas que norman la práctica médica. Las GPC se definen como declaraciones que incluyen recomendaciones destinadas a optimizar la atención al paciente. Se elaboran aplicando revisión sistemática de la evidencia existente, así como la valoración de riesgos y beneficios de las diferentes alternativas de cuidado sanitario disponibles (1). Las GPC han evolucionado en las últimas décadas, desde aquellas elaboradas por opinión basada en experiencia, el consenso de expertos, hasta las GPC desarrolladas con revisión sistemática de evidencias (2). El sistema GRADE para la elaboración de GPC recomienda el uso de la mejor evidencia disponible, balance entre riesgos y beneficios, análisis de factibilidad, uso de recursos de las intervenciones, e incorporación de valores y preferencias de los pacientes, generando recomendaciones con valoración de nivel de certeza, fuerza y dirección (3). Actualmente, la biblioteca de la red internacional de guías clínicas "International Guideline Library" registra un total 6589 documentos en 84 países (4). Además de la elaboración de GPC de novo, se promueve el desarrollo de GPC adaptadas y adoptadas, basadas en GPC de adecuado rigor metodológico que se actualicen y contextualicen incorporando evidencia regional y local (5).

Las GPC brindan el marco referencial para el desempeño del profesional de salud para intervenciones específicas. Cuando las GPC son elaboradas según estándares metodológicos mínimos, garantizan una intervención sanitaria adecuada brindando soporte científico al actuar de los profesionales de la salud con recomendaciones pertinentes y apropiadas (6). Las GPC norman los criterios de atención y manejo de acuerdo con la complejidad de cada intervención. La aplicación regular de las GPC en la práctica clínica orienta al equipo de salud en la toma de decisiones basándose no solo en la mejor evidencia disponible; sino también en la experiencia de facultativos y pacientes (7). Asimismo, el trabajo clínico desarrollado en el marco de las GPC protege al profesional de salud en caso de situaciones controversiales (8).

Las GPC en neurología están diversificadas en todas las subespecialidades y son de uso cada vez más frecuente en la práctica clínica. Existen GPC para muchas enfermedades neurológicas, comunes y poco frecuentes, agudas

Centro de Investigación Básica en Neurogenética, Instituto Nacional de Ciencias Neurológicas. Lima, Perú.

Centro de Salud Global, Universidad Peruana Cayetano Heredia. Lima, Perú.

Facultad de Ciencias de la Salud, Universidad Científica del Sur., Lima, Perú.

Red de Eficacia Clínica y Sanitaria, REDECS. Lima, Perú.

5 Servicio de Neurología, Departamento de Medicina y Oficina de Apoyo a la Docencia e Investigación (OADI), Hospital Daniel Alcides Carrión. Callao, Perú.

6 Servicio de Neurología, Instituto Peruano de Neurociencias. Lima, Perú.

7 Revista de Neuro-Psiquiatria, Facultad de Medicina Alberto Hurtado, Universidad Peruana Cayetano Heredia. Lima, Perú.

a Editor asociado; ${ }^{\mathrm{b}}$ Médico neurólogo; ${ }^{\mathrm{c}}$ Investigador; ${ }^{\mathrm{d}}$ Editor Jefe 
y crónicas desarrolladas principalmente por grupos de trabajo afiliados a organizaciones académico-científicas y médico-institucionales como NICE, SIGN, CENETEC entre otros. En EUA, la Academia Americana de Neurología publica regularmente GPC, cuenta con al menos 204 documentos publicados en 10 categorías diferentes incluyendo ictus y neurología vascular, neuropediatria, enfermedades neuromusculares, epilepsia entre otros (9). En Europa, las guías en neurología son publicadas por varias organizaciones destacando la Academia Europea de Neurología que registra 102 documentos de acceso libre (10). En países de estas regiones las GPC son de uso obligatorio.

La elaboración de GPC para enfermedades neurológicas en el Perú está creciendo significativamente; sin embargo, su aplicación en contexto clínico es aún limitada. En el Perú, la norma técnica vigente para la elaboración de guías de práctica clínica fue publicada el 2015 por el Ministerio de Salud (11); esta norma técnica contempla la elaboración de GPC de acuerdo con el rigor metodológico internacional (GRADE) e incorpora otros aspectos como criterios de referencia y contrareferencia por niveles de atención, poco habituales en GPC internacionales. Existen GPC para enfermedades neurológicas elaboradas en el Ministerio de Salud, EsSalud, Fuerzas Armadas y policiales y práctica privada; sin embargo, la calidad metodológica utilizada es variable entre ellas. Las experiencias de la elaboración de GPC con criterios GRADE con apoyo de la Sociedad Peruana de Neurología para la GPC de Esclerosis Múltiple publicada en este número de la revista, y del Instituto Nacional de Ciencias Neurológicas para la GPC de Síndrome de Guillain Barre (12) confirman la importancia de contar con un soporte institucional y un equipo técnico especializado. El gran reto para los próximos años será seguir promoviendo la elaboración de GPC y su aplicación a nivel nacional. La implementación universal y contextualizada de las GPC requiere políticas de salud y un marco normativo apropiado que promueva su aplicación con supervisión constante y coordinada en cada subsistema de salud en el país.

La elaboración de GPC en neurología en el Perú debe continuar e incrementarse asegurando su implementación y aplicación, evaluación y verificación de resultados y modificación de GPC según necesidades regionales y locales (13). La elaboración de GPC de novo es posible, sobretodo en enfermedades neurológicas de perfil epidemiológico único en el país. La adaptación y contextualización de guías de buena calidad deben realizarse incorporando investigación regional y local para su aplicación en el contexto nacional. El compromiso y participación de los tomadores de decisiones en salud garantizará el cambio de paradigma para mejorar la calidad nuestros servicios de salud.

\section{REFERENCIAS BIBLOGRAFICAS}

1. Institute of Medicine (US) Committee on Standards for Developing Trustworthy Clinical Practice Guidelines. Clinical practice guidelines we can trust. Washington DC: National Academies Press; 2011.

2. Alonso-Coello P, Irfan A, Solà I, Gich I, DelgadoNoguera M, Rigau D, et al. The quality of clinical practice guidelines over the last two decades: a systematic review of guideline appraisal studies. Qual Saf Health Care. 2010;19(6):e58. doi: 10.1136/qshc.2010.042077

3. Guyatt GH, Oxman AD, Vist GE, Kunz R, FalckYtter Y, Alonso-Coello P, et al. GRADE: an emerging consensus on rating quality of evidence and strength of recommendations. BMJ. 2008; 336(7650):924-6. doi: 10.1136/bmj.39489.470347. $\mathrm{AD}$

4. Guidelines International Network. International Guideline Library. Perthshire, Scotland: Guidelines International Network. (Citado el 16 de diciembre del 2019) Disponible en: http://www.g-i-n.net/ library/ international-guidelines-library.

5. Alva-Diaz C, García-Mostajo JA, Gil-Olivares F, Timana R, Pimentel P, Canelo-Aybar C. Guías de práctica clínica: evolución, metodología de elaboración y definiciones actuales. Acta Médica Peruana. 2017;34(4):317-322.

6. Chen Y, Hu S, Wu L, Fang X, Xu W, Shen G. Clinical practice guidelines for hypertension in China: a systematic review of the methodological quality. BMJ Open. 2015;5(7):e008099.

7. Sackett DL, Rosenberg WM, Gray JA, Haynes RB, Richardson WS. Evidence based medicine: what it is and what it isn't. BMJ. 1996;312(7023):71-2. DOI: 10.1136/bmj.312.7023.71

8. Bahtsevani C, Udén G, Willman A. Outcomes of evidence-based clinical practice guidelines: a systematic review. Int $\mathrm{J}$ Technol Assess Health Care. 2004;20(4):427-33.

9. Academia Americana de Neurología. Guidelines. Minnesota, Estados Unidos: Academia Americana de Neurología; 2017.Disponible en: https://www. 
Guías de práctica clínica basadas en evidencia: Desarrollo y aplicación en neurología clínica en el Perú.

aan.com/Guidelines/Home/ByStatusOrType? status $=$ all

10.European Academy of Neurology. Guideline Reference Center. Vienna, Austria: European Academy of Neurology;2018.Disponibleen:https:// www.ean.org/Neurology-Guidelines.2680.0.html

11. Ministerio de Salud. Norma técnica para la elaboración y uso de guías de práctica clínica del Ministerio de Salud. Norma técnica $\mathrm{N}^{\circ}$ 117-MINSA/DGSP-V.01. Resolución Ministerial $N^{\circ}$ 302-2015/MINSA. Lima: Ministerio de Salud; 2015.
12. Alva-Diaz C, Mori N, Pacheco-Barrios K, Velásquez-Rimachi V, Rivera-Torrejon $\mathrm{O}$, Huerta-Rosario CA, et al. Guía de práctica clínica para el diagnóstico y tratamiento del paciente con síndrome de Guillain-Barré, Perú, 2018. Neurología Argentina. 2019; (por publicarse). DOI: 10.1016/j. neuarg.2019.09.006

13. Grimshaw JM, Thomas RE, MacLennan G, Fraser C, Ramsay CR, Vale L, et al. Effectiveness and efficiency of guideline dissemination and implementation strategies. Health Technol Assess. 2004;8(6): 1-72. 